\title{
Quasicomparison Functions and Substructure Synthesis for Framed Structures Stability Analysis
}

\section{Abstract}

The Rayleigh-Ritz-Meirovitch substructure synthesis method (RRMSSM) is extended to buckling analysis in framed structures. The objective is a computational procedure capable of yielding very accurate critical loads through solution of very-low-order eigenvalue problems. In this regard, numerical examples demonstrate that the convergence characteristics of the proposed RRMSSM for stability analysis are superior to those associated with the finite element method.

\section{Keywords}

Quasicomparison, substructure synthesis, structural stability, buckling.

\section{J. I. Colombo ${ }^{\text {a }}$}

C. A. Morales

a Depto. de Mecánica, Universidad Simón Bolívar, Apdo. 89000, Caracas 1080A, Venezuela; colombojose@gmail.com

b Depto. de Mecánica, Universidad Simón Bolívar, Apdo. 89000, Caracas 1080A, Venezuela; cmorales@usb.ve

http://dx.doi.org/10.1590/1679-78252152

Received 20.05.2015

In revised form 20.07.2015

Accepted 13.08.2015

Available online 25.08.2015

\section{INTRODUCTION}

A perpetual goal of Computational Mechanics is the development of approximate methods that produce highly accurate results - critical loads in Structural Stability - and that involve low numbers of degrees of freedom. When the analyst's demands are those both, high accuracy and loworder models, the finite element method is not the most efficient in some Computational Mechanics instances (Meirovitch \& Kwak 1990, Meirovitch \& Kwak 1991, Morales 2000a).

The Rayleigh-Ritz substructure synthesis method (Meirovitch \& Hale 1981) with quasicomparison functions (Meirovitch \& Kwak 1990) was presented compoundly by Meirovitch and Kwak (1991). Several investigations on applications of this methodology to Structural Dynamics have shown the superiority of the convergence characteristics of the technique when it is compared with the finite element method (FEM), and when the structures can be modeled as assemblages of simple substructures (Meirovitch \& Kwak 1991, Morales 2000a, Morales 2000b, Morales 2009). Two reasons for this success of the methodology: 1) the structure is divided into 
its natural and intuitive substructures or superelements and, principally 2) the selection of admissible functions that are related to the dynamics problem being solved and that make up a quasicomparison function. This new technique is referred to as Rayleigh-Ritz-Meirovitch substructure synthesis method (RRMSSM) (Morales 2009).

As it is noted, this RRMSSM has been developed and applied to carry out dynamic analysis, that is, to obtain natural frequencies and modes (eigensolution); because it has been successful in the analysis of some structures such as frames, the question is if it would also be highly efficient in other Structural Mechanics problems involving frames, as stability analysis. It is also noted that the main reason for the achievement is the idea of quasicomparison function (QCF), it has been lately redefined as a linear combination of admissible functions (AF) that is capable of satisfying the complementary boundary conditions (Morales \& Goncalves 2007), the implication is that the AF should be from different families of functions.

In this paper, therefore, extension of Rayleigh-Ritz-Meirovitch substructure synthesis to buckling analysis in frames is investigated. The energy or static approach to stability is considered herein (Bazant \& Cedolin 1991); previously, the vibration or dynamic approach to stability was considered; nonetheless, this methodology (synthesis through the dynamic method) involves two converging processes which make it very cumbersome (Morales \& Ciaccia 2007). The objective of the present work is a computational procedure able to yield very accurate critical loads through solution of very-low-order eigenvalue problems; in other words, to solve the unwieldiness of the dynamic method. It is reported that the goal has been obtained: examples prove that the convergence characteristics of this proposed RRMSSM for buckling analysis, are superior to those associated with the FEM.

Even though the particular literature review on the RRMSSM is straightforward as basically Meirovitch, Morales and collaborators have worked on this method, a related-subject and recent literature review has been conducted. Areny et al. (2011) have also worked on linear structural stability using substructuring; nevertheless, the concentration was on a new uncertainty propagation method based on the concept of lack of knowledge, which comes from the field of Model Updating; interestingly, there is an application of the RRMSSM in Model Updating (Halevi et al., 2005). Rahman and Alam (2014) have dealt with buckling in hybrid piezoelectric beams under electromechanical load; on the other hand, da Mota et al. (2015) have worked on nonlinear stability of piles subjected to bilateral contact constraints underground. In these two cases, the FEM is by far the preferred method; in the first case the coupled zig zag theory is the basis for the FEM. Finally, Sinir et al. (2014) have studied buckling in Euler-Bernoulli beams with elastic supports and considering the von Kármán nonlinearity.

\section{RRMSSM FOR FRAME STABILITY}

The expansion of the RRMSSM to buckling analysis in framed structures is studied. As previously indicated, the RRMSSM has been a dynamic analysis technique; because the static or energy approach to stability is considered in this work (Bazant \& Cedolin 1991), the procedure will have modifications that are studied or will arise in this section. A two-dimensional representative of this type of structures is shown in Fig. 1; nonetheless, the following analysis is valid for three- 
dimensional frames; indeed, more general structures can be negotiated as long as the system can be modeled as an assemblage of simple substructures (Meirovitch \& Kwak 1991, Morales 2000a). The principal concern is the computational efficiency of the method with regard to attainment of the first critical load $P_{c r}$.

The definition of the algebraic (or matrix) buckling eigenvalue problem of a general structure, an approximation to the actual differential eigenvalue problem, requires the derivation of the stiffness matrix of the structure, which can be in turn obtained through its potential energy expression; nonetheless, in the case of frames or multiply supported structures what it is dealt with at the outset is the potential energy of a partially disjoint structure, $V_{d}$; the disjoint structure is afterward fully coupled by enforcing the geometric compatibility conditions that have not been considered.

In the spirit of a generalized Rayleigh-Ritz theory (Meirovitch \& Hale 1981), the elastic deformation vector in a substructure is assumed in the form of a finite series of vectors of spacedependent admissible functions (AF) multiplied by generalized coordinates. Therefore, the substructure deformation field can be expressed as

$$
\mathbf{u}_{s}\left(\mathbf{r}_{s}\right)=\sum_{i=1}^{n_{s}} \boldsymbol{\phi}_{s_{i}}\left(\mathbf{r}_{s}\right) q_{s_{i}}=\boldsymbol{\Phi}_{s}\left(\mathbf{r}_{s}\right) \mathbf{q}_{s} \quad s=1,2, \ldots, N
$$

where $\mathbf{r}_{s}$ is the substructure position vector, $\boldsymbol{\phi}_{s_{i}}$ are 3 -order vectors of $\mathrm{AF}, \boldsymbol{\Phi}_{s}$ is a $3 \times n_{s}$ matrix of $\mathrm{AF}$ and $\mathbf{q}_{s}$ is a vector of substructure generalized coordinates; lastly, $n_{s}$ is the number of substructure coordinates and $N$ is the number of substructures; in vibrations, the coordinates vector is time-dependent, this is the first difference or modification to note in relation to previous work. Now, AF must satisfy certain conditions for each of the 3 components of $\mathbf{u}_{s}$ to qualify as a quasicomparison function $(\mathrm{QCF})$ which in turn insures the extraordinary convergence characteristics previously reported (Meirovitch \& Kwak 1990, Meirovitch \& Kwak 1991, Morales 2000a, Morales 2000b, Morales 2009, Morales \& Goncalves 2007). In fact, each $\boldsymbol{\Phi}_{s}$ row must include functions from different families; more precisely or as indicated in the Introduction, a QCF is a linear combination of AF that is able to satisfy the complementary boundary conditions (Morales \& Goncalves 2007, Baruh \& Tadikonda 1989).

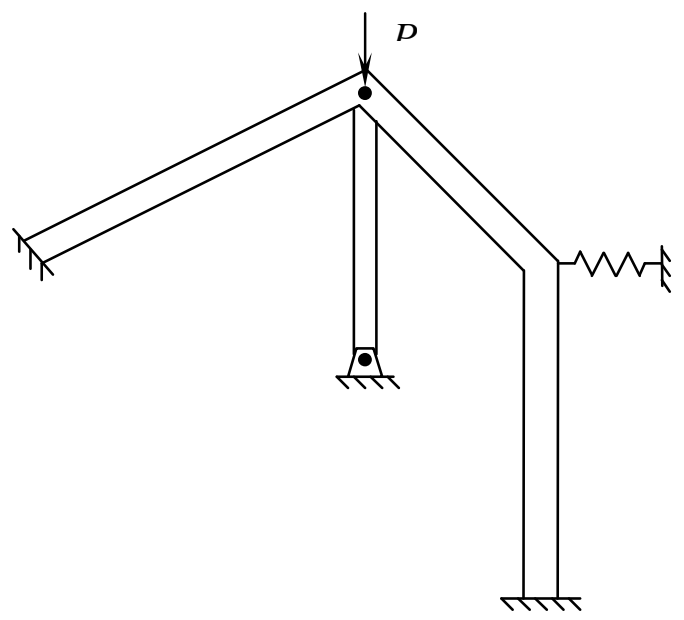

Figure 1: Framed structure under load. 
Now, the QCF vectors $\mathbf{u}_{s}$ are to be introduced into the disjoint potential energy expression $V_{d}$, this lets us write this energy as

$$
V_{d}(P)=\frac{1}{2} \mathbf{q}_{d}^{T} \mathbf{K}_{d}(P) \mathbf{q}_{d}
$$

which brings in the disjoint stiffness matrix $\mathbf{K}_{d}(P)$. Two more fundamental differences between the original RRMSSM for dynamic analysis (Meirovitch \& Kwak 1991, Morales 2000a) and this RRMSSM for stability analysis, first, herein kinetic energies are no longer relevant, secondly, the dependence here of the stiffness matrix on the load $P . \mathbf{q}_{d}$ is the $m$-order disjoint configuration vector,

$$
\mathbf{q}_{d}=\left(\begin{array}{llll}
\mathbf{q}_{1}^{T} & \mathbf{q}_{2}^{T} & \ldots & \mathbf{q}_{N}^{T}
\end{array}\right)^{T}
$$

Next, the stiffness matrix of the assembled or actual structure is obtained by imposing the compatibility conditions not satisfied up to this point in the analysis. These conditions yield linear equations in the components of $\mathbf{q}_{d}$; naturally, if there are $j$ such constraint equations, the assembled structure has finally $n=m$ - $j$ degrees of freedom (DOF). Hence, if $\mathbf{q}$ is the chosen $n$-order vector of independent generalized coordinates of the coupled structure, its relation with the disjoint configuration vector can be written in the form

$$
\mathbf{q}_{d}=\mathbf{C q}
$$

where $\mathbf{C}$ is a sparse $m \times n$ constraint matrix. This equation is introduced into eq. 2 to get the potential energy of the assembled structure, and thus the structure's stiffness matrix as

$$
\mathbf{K}(P)=\mathbf{C}^{T} \mathbf{K}_{d}(P) \mathbf{C}
$$

But in elastic buckling the stiffness matrix, either disjoint or actual, can be separated into elastic and geometric stiffness matrices,

$$
\begin{aligned}
& \mathbf{K}(P)=\mathbf{K}_{e}-\mathbf{K}_{g}(P) \\
& \mathbf{K}_{d}(P)=\mathbf{K}_{d, e}-\mathbf{K}_{d, g}(P)
\end{aligned}
$$

An interesting note is that in the literature there are diverse equivalents for elastic when dealing with $\mathbf{K}_{e}$, material, conventional, standard, linear and even mechanical can be read; in the case of $\mathbf{K}_{g}$ there is not much consensus either, initial-stress, incremental, differential, load-geometric and stability-coefficient are 'synonyms' for geometric.

Finally, recalling that the condition of instability or critical load is (Bazant \& Cedolin 1991, Chajes 1974)

$$
\operatorname{det} \mathbf{K}(P)=0
$$

and that $\mathbf{K}_{g}$ is a linear function of the load $P$, the stability algebraic eigenvalue problem can be written as

$$
\mathbf{K}_{e} \mathbf{q}=P \mathbf{K}_{g}^{*} \mathbf{q}
$$

which is a Rayleigh-Ritz approximation to the actual differential eigenproblem of the framed structure. The eigensolution - critical loads (eigenvalues) and buckling modes- is attained by a converging process in which the number $n_{s}$ of vectors of AF in eq. 1 is increased continuously, for 
each substructure. A main difference between this static approach and the dynamic one (Morales \& Ciaccia 2007) is that in the latter, two distinct converging processes are involved which make it unwieldy; as indicated before, to correct this is an objective of this paper.

\section{APPLICATIONS AND RRMSSM AND FEM CONVERGENCE COMPARISON, RESULTS}

The proposed buckling analysis method is applied to two structures. The simple one is a two-span continuous beam, shown in Fig. 2, it is clamped at one end and simply supported at an intermediate point and the other end. In this case there definitely are two natural and intuitive substructures or superelements. As explained in the theoretical or last Section, the first part of the procedure is the derivation of the structure's potential energy. It is just easier to start with a disjoint structure potential energy,

$$
V_{d}=\frac{1}{2}\left(\int_{0}^{L_{1}} E I\left(\frac{d^{2} y_{1}}{d x_{1}^{2}}\right)^{2}-P\left(\frac{d y_{1}}{d x_{1}}\right)^{2} d x_{1}+\int_{0}^{L_{2}} E I\left(\frac{d^{2} y_{2}}{d x_{2}^{2}}\right)^{2}-P\left(\frac{d y_{2}}{d x_{2}}\right)^{2} d x_{2}\right)
$$

where $L_{1}$ and $L_{2}$ are the lengths of the two spans, $E I$ is the flexural rigidity of the beam and $y_{1}$ and $y_{2}$ are the substructures elastic deformations. The local rigid-body axes, $x_{1}$ and $x_{2}$, over which elastic deformation occurs, are shown in Fig. 3.

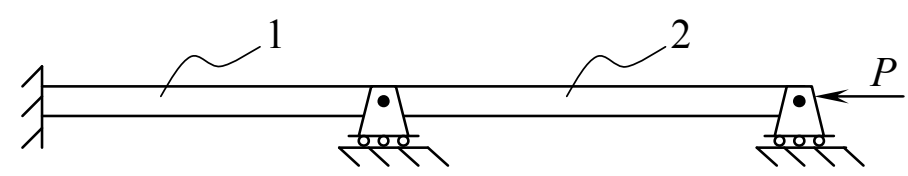

Figure 2: Loaded two-span continuous beam.

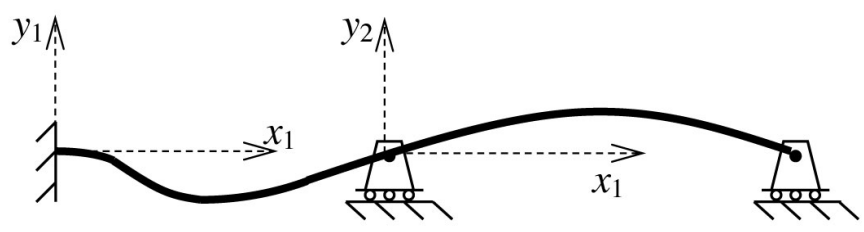

Figure 3: Deformed beam.

Next, the elastic deformation of each substructure is expressed in terms of quasicomparison functions $(\mathrm{QCF})$ as follows

$$
y_{1}\left(x_{1}\right)=\mathbf{f}^{T}\left(x_{1}\right) \mathbf{q}_{1} \quad y_{2}\left(x_{2}\right)=\mathbf{g}^{T}\left(x_{2}\right) \mathbf{q}_{2}
$$

where $\mathbf{f}$ and $\mathbf{g}$ are vectors of admissible functions (AF); recall that AF satisfy only geometric boundary conditions (Meirovitch 1997); in the case of frames, substructural AF should satisfy the substructure geometric boundary conditions (GBC). Now, it was pointed out that selected AF must be related to the mechanics problem under consideration; thus, in the case of frame buckling beam-column eigenfunctions represent an unbeatable alternative; more importantly, if AF are to 
be combined into a QCF, this linear combination must be capable of satisfying the complementary boundary conditions (CBC) (Morales \& Goncalves 2007).

A thorough explanation of the function families selection process is necessary. First, the primary and inviolable substructural GBC are:

$$
\begin{array}{rcr}
y_{1}(0)=0 & \frac{d y_{1}}{d x_{1}}(0)=0 & y_{1}\left(L_{1}\right)=0 \\
y_{2}(0)=0 & y_{2}\left(L_{2}\right)=0 &
\end{array}
$$

Thus, in the case of the first beam the basic or admissibility requisite demands that each family making up the QCF be characterized by a clamped left end; notice that the other standard beam end conditions: free, pinned and sliding, do not satisfy both GBC. At the other boundary, $x_{1}=L_{1}$, there is only one GBC to be satisfied, and this can be done with either a pinned or clamped boundary. Because the QCF concept demands at least or just two families of functions, the problem for the first beam seems solved; it is, the final word or reason is that the mixture of clampedpinned plus clamped-clamped is able to satisfy the CBC which can be expressed as

$$
\frac{d^{2} y_{1}}{d x_{1}^{2}}(0) \neq 0 \quad \frac{d^{3} y_{1}}{d x_{1}^{3}}(0) \neq 0 \quad \frac{d y_{1}}{d x_{1}}\left(L_{1}\right) \neq 0 \quad \frac{d^{2} y_{1}}{d x_{1}^{2}}\left(L_{1}\right) \neq 0 \quad \frac{d^{3} y_{1}}{d x_{1}^{3}}\left(L_{1}\right) \neq 0
$$

It has to be emphasized that the combination should just have the freedom to satisfy the CBC; it is not that the each function must satisfy all CBC, that is for GBC.

For the second beam, mere AF are defined by eq. 12 which imply that each family must be either pinned or clamped at each edge. Now and again, a genuine QCF or combination of two families of AF must be able to allow for the $\mathrm{CBC}$ to be satisfied, which are:

$$
\frac{d y_{2}}{d x_{2}}(0) \neq 0 \quad \frac{d^{2} y_{2}}{d x_{2}^{2}}(0) \neq 0 \quad \frac{d^{3} y_{2}}{d x_{2}^{3}}(0) \neq 0 \quad \frac{d y_{2}}{d x_{2}}\left(L_{2}\right) \neq 0 \quad \frac{d^{3} y_{2}}{d x_{2}^{3}}\left(L_{2}\right) \neq 0
$$

Because the fundamental GBC imply that there are only four possible families: pinned-pinned, pinned-clamped, clamped-pinned and clamped-clamped, 14c and 14e are disregarded because they are satisfied by any function of this remaining set. Now, at the left end we must have one AF pinned and the other clamped so that the QCF allows the possibility of the inequalities 14a and 14b, respectively. Finally, at the right boundary both functions cannot be clamped because there would not be capability of satisfying $14 \mathrm{~d}$; consequently, the possible pairs of AF or combinations are: pinned-pinned plus clamped-clamped, pinned-clamped plus clamped-pinned and pinnedpinned plus clamped-pinned. The last couple of families is chosen because it perfectly satisfies

$$
\frac{d^{2} y_{2}}{d x_{2}^{2}}\left(L_{2}\right)
$$

this is neither a GBC or a CBC, which are the sole preoccupations in the Rayleigh-RitzMeirovitch method, but if we have the freedom or chance to satisfy it, one should take advantage of it as another type of worry is convergence speed. A more intuitive and less mechanistic reason is "because the actual end of the structure is pinned". It could be argued that there is another GBC that was not considered around eqs. 11 and 12; indeed, the other GBC is: 


$$
\frac{d y_{1}}{d x_{1}}\left(L_{1}\right)=\frac{d y_{2}}{d x_{2}}(0)
$$

Nonetheless, this is an example of constraint equations (previous theory) which are different from substructure GBC in that two or more substructures are involved, there is impossibility of being satisfied at the outset as consequence; nevertheless, this equation is considered in a necessary and subsequent constraining process as explained in Section 2. Regardless of this, the quasicomparison functions, eqs. 10, are conclusively defined through

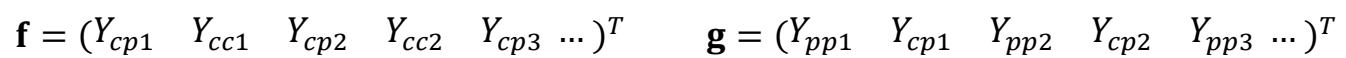

where $Y_{c p}, \quad Y_{c c}$ and $Y_{p p}$ are the clamped-pinned, clamped-clamped and pinned-pinned beamcolumn eigenfunctions (Colombo \& Morales 2012a).

Next, substituting eqs. 10 into eq. 9, the disjoint potential energy can be expressed as

$$
V_{d}=\frac{1}{2}\left(\int_{0}^{L_{1}} E I\left(\mathbf{f}^{\prime \prime T} \mathbf{q}_{1}\right)^{2}-P\left(\mathbf{f}^{\prime T} \mathbf{q}_{1}\right)^{2} d x_{1}+\int_{0}^{L_{2}} E I\left(\mathbf{g}^{\prime \prime T} \mathbf{q}_{2}\right)^{2}-P\left(\mathbf{g}^{\prime T} \mathbf{q}_{2}\right)^{2} d x_{2}\right)
$$

or as eq. 2 after writing the various quadratic forms in matrix notation and integrating. Advantageous, closed-form and interestingly simple results for the integrals involved have been obtained in this same research project and released earlier (Colombo \& Morales 2012a).

Finally, the geometric compatibility conditions not satisfied up to this point in the analysis must be enforced by a constraining process. The slope compatibility between the beams at $x_{1}=L_{1}$ $\left(x_{2}=0\right)$ has not been taken into account yet, that implies that the beams remain at a zero angle with respect to each other at that joint; the mathematical expression of this compatibility condition is the constraint equation 16. Inserting eqs. 10 into the constraint equation leads to

$$
\mathbf{f}^{\prime T}\left(L_{1}\right) \mathbf{q}_{1}=\mathbf{g}^{\prime T}(0) \mathbf{q}_{2}
$$

which can be used to define the constraint matrix $\mathbf{C}$ by means of eq. 4 ; at the end, the assembled-structure stiffness matrix is defined through eq. 5 .

To corroborate the effectiveness of the proposed synthesis method for structural stability, we consider a continuous steel $\left(E=2 \times 10^{11} \mathrm{~Pa}\right)$ beam with the following characteristics: $L_{1}=2.6 \mathrm{~m}$, $L_{2}=2 \mathrm{~m}$ and $I=8.333 \times 10^{-6} \mathrm{~m}^{4}$. The buckling problem is solved by both, the RRMSSM and the FEM; in the last case, beam elements with 2 DOF per node and cubic polynomials as interpolation functions have been used; furthermore, we have a third approximate solution for the beam with this geometry (Bazant \& Cedolin 1991). The convergence of the first critical load to 4 digits is shown in Table 1.

It can be concluded that, for a given accuracy, the solution yielded by the RRMSSM requires appreciably fewer DOF than a solution produced by the FEM; to be specific, 4-digit convergence is achieved with just 7 DOF by means of the synthesis method; in contrast, thru the application of the FEM that accuracy level is achieved with 50 DOF. The other or third approximate method mentioned above may lead to erroneous loads in some cases, as discussed in the reference (Bazant \& Cedolin 1991). 
The other studied structure is the symmetrically-loaded portal frame such as the one shown in Fig. 4. Naturally and intuitively, there are 3 substructures in this case. A disjoint potential energy, of an L-shaped structure plus a cantilever column, is

$$
\begin{aligned}
V_{d}= & \frac{1}{2}\left(\int_{0}^{L} E I_{c}\left(\frac{d^{2} y_{1}}{d x_{1}^{2}}\right)^{2}-P\left(\frac{d y_{1}}{d x_{1}}\right)^{2} d x_{1}+\int_{0}^{l} E I_{b}\left(\frac{d^{2} y_{2}}{d x_{2}^{2}}\right)^{2} d x_{2}\right. \\
& \left.+\int_{0}^{L} E I_{c}\left(\frac{d^{2} y_{3}}{d x_{3}^{2}}\right)^{2}-P\left(\frac{d y_{3}}{d x_{3}}\right)^{2} d x_{3}\right)
\end{aligned}
$$

where $L$ and $E I_{c}$, and $l$ and $E I_{b}$ are the length and flexural rigidity of the columns and beam, respectively; the local rigid-body axes, $x_{i}$, are shown in Fig. 5. The elastic deformations are expressed in terms of QCF as follows

\begin{tabular}{|c|c|c|}
\hline \multirow[t]{2}{*}{$n$} & \multicolumn{2}{|c|}{$P[\mathrm{kN}]$} \\
\hline & RRMSSM & FEM \\
\hline 1 & 450.6 & \\
\hline 2 & & 578.2 \\
\hline 3 & 447.8 & \\
\hline 5 & 447.7 & \\
\hline 6 & & 454.2 \\
\hline 7 & 447.6 & \\
\hline 9 & 447.6 & \\
\hline 10 & & 449.1 \\
\hline 14 & & 448.1 \\
\hline 18 & & 447.8 \\
\hline 22 & & 447.7 \\
\hline 26 & & 447.7 \\
\hline 30 & & 447.7 \\
\hline 34 & & 447.7 \\
\hline 38 & & 447.7 \\
\hline 42 & & 447.7 \\
\hline 46 & & 447.7 \\
\hline 50 & & 447.6 \\
\hline
\end{tabular}

$$
y_{1}\left(x_{1}\right)=\mathbf{f}^{T}\left(x_{1}\right) \mathbf{q}_{1} \quad y_{2}\left(x_{2}\right)=\mathbf{g}^{T}\left(x_{2}\right) \mathbf{q}_{2} \quad y_{3}\left(x_{3}\right)=\mathbf{f}^{T}\left(x_{3}\right) \mathbf{q}_{3} \quad(21 \mathrm{a}, \mathrm{b})
$$

Table 1: Continuous beam critical load convergence. 


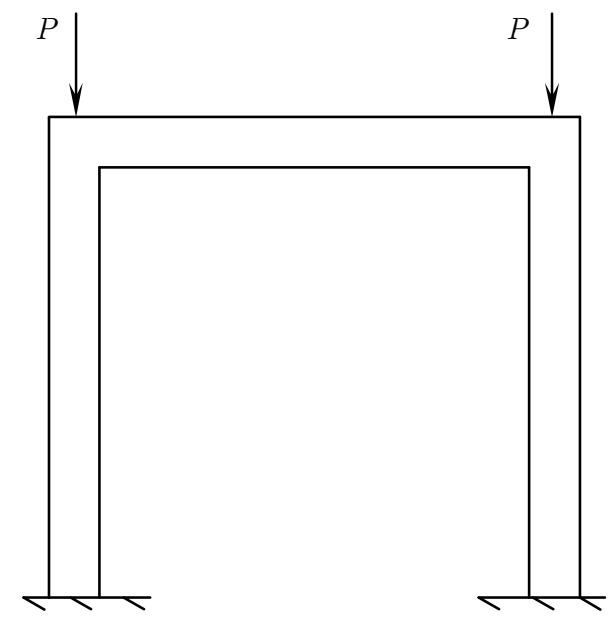

Figure 4: Loaded portal frame.

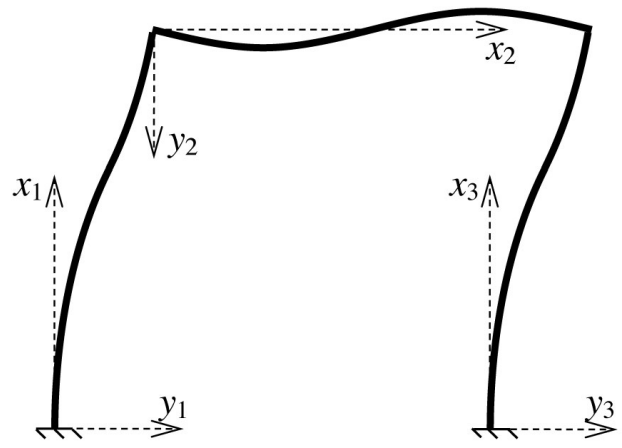

Figure 5: Deformed portal frame.

In this case, the untouchable and primary substructural GBC are:

$$
\begin{array}{ll}
y_{1}(0)=0 & \frac{d y_{1}}{d x_{1}}(0)=0 \\
y_{2}(0)=0 & y_{2}(l)=0 \\
y_{3}(0)=0 & \frac{d y_{3}}{d x_{3}}(0)=0
\end{array}
$$

Consequently, the admissibility requisite demands, in the case of both columns, that each family forming the QCF be characterized by a clamped left end. At the 'right' end, $x_{1(3)}=L$, there are not GBC to be satisfied. Now, the QCF concept requires that the linear combination of (at least) two families be capable of satisfying the CBC, which for column 1 can be expressed as

$$
\frac{d^{2} y_{1}}{d x_{1}^{2}}(0) \neq 0 \quad \frac{d^{3} y_{1}}{d x_{1}^{3}}(0) \neq 0 \quad y_{1}(L) \neq 0 \quad \frac{d y_{1}}{d x_{1}}(L) \neq 0 \quad \frac{d^{2} y_{1}}{d x_{1}^{2}}(L) \neq 0 \quad \frac{d^{3} y_{1}}{d x_{1}^{3}}(L) \neq 0
$$


(column 2 has the same CBC). Notice that the first 2 conditions are already satisfied if, as established, the $\mathrm{AF}$ are clamped at $x_{1(3)}=0$; more importantly, note that at the other edge, the combination must be capable of allowing for nonzero displacement, slope, bending moment and shearing force. Therefore, there are only 2 correct possibilities for the mixture: clamped-free plus clamped-clamped and clamped-sliding plus clamped-pinned; the first combination is chosen herein, for both columns.

It must be pointed out that even though we have analyzed this structure in a previous regional conference paper (Colombo \& Morales 2012b), the thorough explanation of the admissible function selection process is missing therein; it should be stressed that this function families selection process is the most important and interesting part of the substructure synthesis method as it is a part that cannot be done by a computer or program, it is the work of the dynamicist who has to choose optimal families of functions for the particular structure involved; furthermore, how to choose correct AF is important for anybody who wishes to learn the SSM methodology. For this reasons, herein the full AF selection process is presented.

For the cross-beam, bare AF are defined by eqs. 23 which imply that each family must be either pinned or clamped at each boundary. Again, a genuine QCF must allow the possibility, in this case, of nonzero slope, moment and force at both ends; of course, each of these conditions is connected with one of the substructure CBC, which can be written as

$$
\frac{d y_{2}}{d x_{2}}(0) \neq 0 \quad \frac{d^{2} y_{2}}{d x_{2}^{2}}(0) \neq 0 \quad \frac{d^{3} y_{2}}{d x_{2}^{3}}(0) \neq 0 \quad \frac{d y_{2}}{d x_{2}}(l) \neq 0 \quad \frac{d^{2} y_{2}}{d x_{2}^{2}}(l) \neq 0 \quad \frac{d^{3} y_{2}}{d x_{2}^{3}}(l) \neq 0 \quad(26 \mathrm{a}-\mathrm{f})
$$

where $26 \mathrm{c}$ and $26 \mathrm{f}$ are already satisfied. It can be concluded that there are 2 correct possibilities for the combination: clamped-clamped plus pinned-pinned and pinned-clamped plus clampedpinned. The QCF is chosen as the second option; this choice is based on results for vibrations of a portal frame mounted on springs where clamped-free plus free-clamped proved to deliver a superior frequency solution than clamped-clamped plus free-free (Meirovith \& Kwak 1991). In this regard, we remark that diversity is what makes QCF much more powerful than plain AF; particularly, a combination of pinned-clamped plus clamped-pinned is more diverse than one of clamped-clamped plus pinned-pinned. The QCF are finally defined through

$$
\mathbf{f}=\left(\begin{array}{llllllllllll}
Y_{c f 1} & Y_{c c 1} & Y_{c f 2} & Y_{c c 2} & Y_{c f 3} & \cdots
\end{array}\right)^{T} \quad \mathbf{g}=\left(\begin{array}{llllll}
Y_{p c 1} & Y_{c p 1} & Y_{p c 2} & Y_{c p 2} & Y_{p c 3} & \cdots
\end{array}\right)^{T}
$$

where $Y_{c f}$ and $Y_{p c}$ are the clamped-free and pinned-clamped beam-column eigenfunctions (Colombo \& Morales 2012a). Finally, the constraint equations in this case are

$$
y_{1}(L)=y_{3}(L) \quad \frac{d y_{1}}{d x_{1}}(L)=\frac{d y_{2}}{d x_{2}}(0) \quad \frac{d y_{3}}{d x_{3}}(L)=\frac{d y_{2}}{d x_{2}}(l)
$$

For brevity, in this second example the expected substitution of eqs. 21 into eqs. 20 and 28 is not shown.

A fully converging eigensolution can be obtained for a wide range of frame geometry; for comparison purposes a $4 \mathrm{~m} \times 4 \mathrm{~m}$ steel frame is considered $\left(I=8.333 \times 10^{-6} \mathrm{~m}^{4}\right)$ (Morales \& Ciaccia 2007). The eigenproblem is solved by the RRMSSM and the FEM; again, the convergence of the 
first critical load to 4 digits is shown, in Table 2. The fact of the previous example is confirmed, that the solution produced by the RRMSSM requires appreciably fewer DOF than the solution yielded by the FEM; in this case, 4-digit convergence is attained with 15 DOF by means of the synthesis method, whereas through the application of the FEM the same accuracy is achieved with 27 DOF.

Now, the same frame was analyzed by the vibration or dynamic approach to stability; nonetheless, that methodology (synthesis through the dynamic method) involves two converging processes which make it very cumbersome (Morales \& Ciaccia 2007). Moreover, 21 DOF are required for just 3-digit convergence of the critical load; besides, Euler-Bernoulli or vibration beam functions were used in that previous work as AF, not beam-column or buckling functions as in the present one. Regarding this inferiority in convergence characteristics of the original dynamic method, another example can be reported, it is the inverted-Y structure with clamped lower ends and pinned upper end that is loaded at this edge. The analyzed inverted-Y structures is composed by 3 identical beams separated by $120^{\circ}$, as shown in Fig. 6 ; steel $\left(m=580 \mathrm{~kg} / \mathrm{m}, E I=1.3 \times 10^{8} \mathrm{~N}\right.$ $\mathrm{m}^{2}$ ) columns are considered with lengths $L=5 \mathrm{~m}$. The critical load was attained by the dynamics-RRMSSM as $P_{c r}=73,83 \mathrm{MN}$ but it is not as direct as with the statics-RRMSSM: natural frequency convergence (which is required before $P_{c r}$ convergence can be called) was very difficult to attain as more than 20 DOF again are necessary but even then full or absolute convergence of the natural frequency was elusive. This is because as more AF are used or added into the QCF, linear-dependence and numerical problems arise (Ramírez 2002).

\begin{tabular}{ccc}
\hline \hline$n$ & \multicolumn{2}{c}{$P[\mathrm{kN}]$} \\
\cline { 2 - 3 } & RRMSSM & FEM \\
\hline 3 & 1536 & 775.5 \\
6 & 794,0 & \\
9 & 774.0 & 771.0 \\
12 & 769.4 & \\
15 & 768.7 & 769.2 \\
18 & 768.7 & \\
21 & & 768.8 \\
27 & & 768.7 \\
33 & & 768.7 \\
\hline \hline
\end{tabular}

Table 2: Portal frame critical load convergence.

Therefore, the substructure synthesis through the static approach proposed herein is a genuine advance. This new method can be applied to more complex frames; in fact, the RRMSSM has been applied as successfully in dynamic analysis of more elaborate structures (Morales 2000b). 


\section{CONCLUSIONS}

Rayleigh-Ritz based substructure synthesis was expanded to static-approach buckling analysis of frames. Examples proved that the computational characteristics of the method are superior to those of the standard FEM and the dynamics substructure synthesis method; as consequence, very accurate critical loads can be obtained thru routines characterized by very-low-order stiffness matrices.

As indicated, the energy or static approach to stability was considered; previously, the vibration or dynamic approach to stability had been considered; nevertheless, this methodology (synthesis through the dynamic method) involved two converging processes which made it very cumbersome.

There are two reasons for the success of the proposed method: 1) the structure is divided into its natural and intuitive substructures or superelements and, mainly 2) the selection of admissible functions that are related to the stability problem being solved and that make up a quasicomparison function.

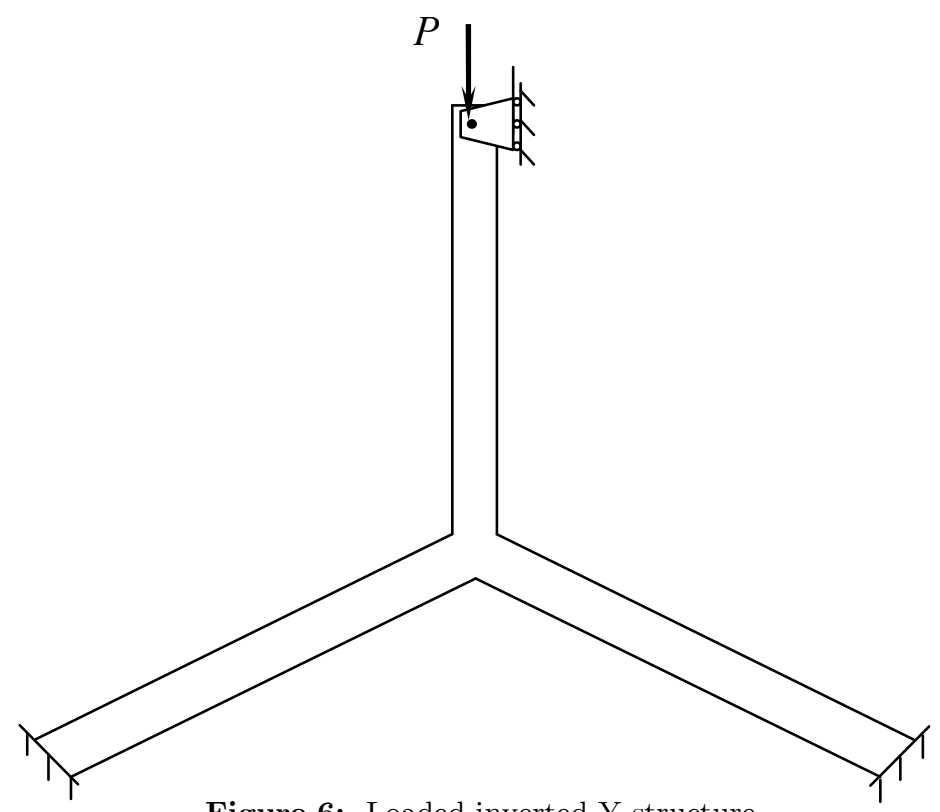

Figure 6: Loaded inverted-Y structure.

\section{Acknowledgements}

This work was supported by Decanato de Investigación, Univ. Simón Bolívar, grant S1-IC-CAI-018-14.

\section{References}

Areny, P., Chambon, L., Cresta, P. (2011). The lack-of-knowledge (LOK) theory for linear buckling analysis problems. Computers and Structures 89:795-800. 
Baruh, H., Tadikonda, S. S. K. (1989). Another look at admissible functions. Journal of Sound and Vibration 132:73-87.

Bazant, Z. P, Cedolin, L. (1991).Stability of Structures, Oxford University Press, New York.

Chajes, A. (1974). Principles of structural stability theory, Prentice-Hall, Englewood Cliffs.

Colombo, J. I., Morales, C. A. (2012a). Simplest-expression integrals involving beam-column eigenmodes. Archives of Mechanics 64:529-535.

Colombo, J. I., Morales, C. A. (2012b). Quasicomparison functions and substructure synthesis for buckling analysis in frames. VII Congreso Bolivariano de Ing. Mecánica, Cusco, Perú.

da Mota, R. A. et al. (2015). Nonlinear equilibrium and stability analysis of axially loaded piles under bilateral contact constraints. Latin American Journal of Solids and Structures 12:250-270.

Halevi, Y., Morales, C. A., Inman, D. J. (2005). Combined expansion and orthogonalization of experimental modeshapes. Journal of Vibration and Acoustics 127:188-196.

Meirovitch, L., Hale, A. L. (1981). On the substructure synthesis method. AIAA Journal 19:940-947.

Meirovitch, L., Kwak, M. K. (1990). Convergence of the classical Rayleigh-Ritz method and the finite element method. AIAA Journal 28:1509-1516.

Meirovitch, L., Kwak, M. K. (1991). Rayleigh-Ritz based substructure synthesis for flexible multibody systems. AIAA Journal 29:1709-1719.

Meirovitch, L. (1997). Principles and techniques of vibrations, Prentice-Hall, Upper Saddle River.

Morales, C. A. (2000a). Rayleigh-Ritz based substructure synthesis for multiply supported structures. Journal of Vibration and Acoustics 122:2-6.

Morales, C. A. (2000b). Dynamic analysis of frames by a Rayleigh-Ritz based substructure synthesis method. Engineering Structures 22:1632-1640.

Morales, C. A. (2009). Dynamic analysis of an L-shaped structure by Rayleigh-Ritz substructure synthesis method. Meccanica 44:339-343.

Morales, C. A., Goncalves, R. (2007). Eigenfunction convergence of the Rayleigh-Ritz-Meirovitch method and the FEM. Shock and Vibration 14:417-428.

Morales, C. A., Ciaccia, M. A. (2007). Substructure synthesis method for buckling analysis in frames. Meccanica 42:601-607.

Rahman, N., Alam, N. M. (2014). Finite element modeling for buckling analysis of hybrid piezoelectric beam under electromechanical loads. Latin American Journal of Solids and Structures 11:770-789.

Ramírez, J. F. (2002). Autosolución de problemas de pandeo y conducción de calor mediante síntesis subestructural. M.Sc thesis (in Spanish), Universidad Simón Bolívar, Venezuela.

Sinir, B. G., Ozhan, B. B., Reddy, J. N. (2014). Buckling configurations and dynamic response of buckled eulerbernoulli beams with non-classical supports. Latin American Journal of Solids and Structures 11:2516-2536. 